\title{
Retrotransposing Gremlins May Disrupt Our Brain's Genomes
}

Human geneticists commonly attribute individual genetic variation to alterations in the germline, thus implicitly assuming that the genome of the zygote is faithfully replicated during the multiple cell divisions needed to produce an adult human being. During DNA replication, errors do arise and are presumed to provoke malignant cell transformation and cellular senescence, which eventually may result in cancer and aging. Also in neurodevelopmental and neurodegenerative disorders, which are in part due to inherited and de novo genome alterations in the germline, somatic genome variation may be involved [Girirajan and Eichler, 2009; State and Levitt, 2011]. Early molecular cytogenetic investigation at the single-cell level revealed that the human developing brain consists of a mosaic of euploid and aneuploid neural cells [Yurov et al., 2007; Iourov et al., 2008]. The average frequency of aneuploidy ranges from 1.25 to $1.45 \%$ per chromosome, and overall, the fraction of aneuploid cells approaches $30-35 \%$. In brains from patients with ataxia telangiectasia, a 5- to 20 -fold increase of nonrandom DNA doublestrand breaks (DSBs) and aneuploidy was found, which may mediate the cerebellar degeneration in this disorder [Iourov et al., 2009]. In actively transcribing cells, such as B lymphocytes and primary neural stem/progenitor cells, the vicinity of transcription start sites of active genes is enriched for DSBs and translocation breakpoints [Schwer et al., 2016]. The junctions of transcription start sites-associated translocations showed signatures of classical and

\section{KARGER}

๑) 2016 S. Karger AG, Basel

E-Mail karger@karger.com

www.karger.com/msy alternative nonhomologous end joining. These DSB response pathways depend on ATM, a gene mutated in ataxia telangiectasia patients who develop immunodeficiency and cerebellar degeneration [Poot and Haaf, 2015]. In inducible pluripotent stem cells from healthy individuals and in brains from schizophrenia patients, mosaic copy number variations (CNVs) disrupting neuronal genes were found [McConnell et al., 2013; Sakai et al., 2015]. In neural stem/progenitor cells, long neural genes were particularly susceptible to recurrent DSB clusters [Wei et al., 2016]. This indicates that DSB response mechanisms are not only required to prevent CNVs and complex chromosome rearrangements in the germline, but also to maintain genome integrity, for instance in the brain [Yurov et al., 2007, 2009; Poot and Haaf, 2015].

Recent studies indicate that yet another mechanism may cause mosaic genome disruption in the human brain. Retrotransposition of DNA segments flanked by long interspersed elements (LINEs) may mediate CNV by nonallelic homologous recombination (NAHR) [Beck et al., 2010]. The human genome contains some 17,000 LINEs spaced less than $10 \mathrm{Mb}$ apart and probably involved in 516 germline CNVs found by diagnostic array CGH of 36,285 patients [Startek et al., 2015]. Analyses of sorted single cells from the human cortex and caudate nucleus showed a LINE-mediated CNV rate of 1/10,000-1/100 events per human neural progenitor cell [Evrony et al., 2012]. This suggests that somatic retrotransposition me- 
diated by L1 type LINE elements in the human cortex and caudate nucleus is a rare event [Evrony et al., 2012]. However, this conclusion is controversial. Single-cell retrotransposon capture-based sequencing of individual human hippocampal neurons and glia cells showed an estimated 13.7 somatic L1 insertions per hippocampal neuron [Upton et al., 2015]. These L1 insertions were specifically enriched in transcribed neuronal stem cell enhancers and hippocampal genes, which suggests that they are probably relevant for neuronal functions. In view of these findings, Evrony et al. [2016] reanalyzed their data and found approximately 0.2 retrotransposition events per cell, which is a lower rate than reported, but still confirms that L1 elements may be mobile in some human neurons [Upton et al., 2015].

Erwin et al. [2016] extracted nuclei from the frontal cortex and the hippocampus of 3 healthy individuals and isolated neuronal nuclei by fluorescence-activated cell sorting after immunostaining for the neural-specific antigen NeuN [Erwin et al., 2016]. The DNA of these single nuclei was amplified by multiple displacement amplification and subsequently subjected to paired-end sequencing. Reads with one end spanning a junction between an L1 element and the flanking genomic sequence were selected and further examined. With this approach, the authors discovered somatic L1-associated variants (SLAVs), which were composed of either retrotransposition-mediated insertions or of retrotransposition-independent L1associated variants. A subset of the SLAVs comprises somatic deletions generated by L1 endonuclease activity. Retrotransposition-independent rearrangements in inherited L1 elements resulted in a deletion of proximal genomic regions. These rearrangements were resolved by microhomology-mediated DNA repair. This suggests that L1-associated genomic regions are hotspots for somatic $\mathrm{CNVs}$ in the brain and, therefore, contribute to somatic genome mosaicism. In hindsight, one could surmise that some of the structural variations detected in previous studies may have been caused by L1 retrotranspositionmediated insertions, but the available technology at this time did not allow to rigorously prove this [Yurov et al., 2007; Iourov et al., 2009; McConnell et al., 2013; Sakai et al., 2015] SLAVs were found in crucial neuronal genes, such as DLG2, which has previously been associated with schizophrenia, and affect $44-63 \%$ of the cells in the healthy human brain. In this way, this study indicates that L1mediated retrotransposition may be a serious cause of mosaic structural variation in the human brain.

The scope of retrotranspositions in the human genome may be even wider than hitherto thought. Mapping the integration sites of L1, Alu and SVA-related germline mutations with a high-throughput retrotransposon capture sequencing protocol, Baillie et al. [2011] found 7,743 putative somatic L1 insertions as well as an additional 13,692 somatic Alu and 1,350 somatic SVA insertions in the hippocampus and caudate nucleus of 3 individuals. These results demonstrate that retrotransposons mobilize to protein-coding genes that are differentially expressed and metabolically active in the brain. The genetic networks underlying normal and abnormal neurobiological processes may thus be reshaped by retrotransposition-driven somatic genome mosaicism [Baillie et al., 2011]. Upon analysis of 8,943 deletion breakpoints in 1,092 whole genome sequences, some $25 \%$ proved to be mediated by transposable element insertions, in particular Alu elements [Abyzov et al., 2015]. Those breakpoints were associated with evolutionarily less-conserved regions and 5 times more likely to reside in hypomethylated regions than expected by chance. This suggests preferential transposable element insertions in transcriptionally active chromatin regions. Finally, in a family in which a chromothripsis event was transmitted among 11 healthy individuals from 3 generations, one inserted SVAE retrotransposon, associated with a $110-\mathrm{kb}$ deletion, and 4 Alu elements flanking a $110-\mathrm{kb}$ deletion and associated with an inversion were reported [Nazaryan-Petersen et al., 2016]. These recent studies open a window to novel transcription-related and retrotransposition-based mechanisms of structural variation in somatic tissues, in particular the brain. They may also prompt further research aiming to comprehensively grasp their possible significance for neurodevelopmental and other disorders.

Martin Poot
References

Mol Syndromol 2017;8:55-57 DOI: $10.1159 / 000453247$
Abyzov A, Li S, Kim DR, Mohiyuddin M, Stütz $\mathrm{AM}$, et al: Analysis of deletion breakpoints from 1,092 humans reveals details of mutation mechanisms. Nat Commun 6:7256 (2015).

Baillie JK, Barnett MW, Upton KR, Gerhardt DJ, Richmond TA, et al: Somatic retrotransposition alters the genetic landscape of the human brain. Nature 479:534-537 (2011).

Beck CR, Collier P, Macfarlane C, Malig M, Kidd JM, et al: LINE-1 retrotransposition activity in human genomes. Cell 141:1159-1170 (2010).

Erwin JA, Paquola AC, Singer T, Gallina I, Novotny $\mathrm{M}$, et al: $\mathrm{L} 1$-associated genomic regions are deleted in somatic cells of the healthy human brain. Nat Neurosci 19:1583-1591 (2016).
56 
Evrony GD, Cai X, Lee E, Hills LB, Elhosary PC, et al: Single-neuron sequencing analysis of $\mathrm{L} 1$ retrotransposition and somatic mutation in the human brain. Cell 151:483-496 (2012).

Evrony GD, Lee E, Park PJ, Walsh CA: Resolving rates of mutation in the brain using singleneuron genomics. Elife 5:e12966 (2016).

Girirajan S, Eichler EE: Phenotypic variability and genetic susceptibility to genomic disorders. Hum Mol Genet 19:R176-187 (2009).

Iourov IY, Vorsanova SG, Yurov YB: Molecular cytogenetics and cytogenomics of brain diseases. Curr Genomics 9:452-465 (2008).

Iourov IY, Vorsanova SG, Liehr T, Kolotii AD, Yurov YB: Increased chromosome instability dramatically disrupts neural genome integrity and mediates cerebellar degeneration in the ataxia-telangiectasia brain. Hum Mol Genet 18:2656-2669 (2009)
McConnell MJ, Lindberg MR, Brennand KJ, Piper JC, Voet T, et al: Mosaic copy number variation in human neurons. Science 342:632637 (2013).

Nazaryan-Petersen L, Bertelsen B, Bak M, Jønson L, Tommerup N, et al: Germline chromothripsis driven by L1-Mmediated retrotransposition and Alu/Alu homologous recombination. Hum Mutat 37:385-395 (2016).

Poot M, Haaf T: Mechanisms of origin, phenotypic effects and diagnostic implications of complex chromosome rearrangements. Mol Syndromol 6:110-134 (2015).

Sakai M, Watanabe Y, Someya T, Araki K, Shibuya $\mathrm{M}$, et al: Assessment of copy number variations in the brain genome of schizophrenia patients. Mol Cytogenet 8:46 (2015).

Schwer B, Wei PC, Chang AN, Kao J, Du Z, et al: Transcription-associated processes cause DNA double-strand breaks and translocations in neural stem/progenitor cells. Proc Natl Acad Sci USA 113:2258-2263 (2016).
Startek M, Szafranski P, Gambin T, Campbell IM, Hixson P, et al: Genome-wide analyses of LINE-LINE-mediated nonallelic homologous recombination. Nucleic Acids Res 43: 2188-2198 (2015).

State MW, Levitt P: The conundrums of understanding genetic risks for autism spectrum disorders. Nat Neurosci 14:1499-1506 (2011).

Upton KR, Gerhardt DJ, Jesuadian JS, Richardson SR, Sánchez-Luque FJ, et al: Ubiquitous L1 mosaicism in hippocampal neurons. Cell 161: 228-239 (2015).

Wei PC, Chang AN, Kao J, Du Z, Meyers RM, et al: Long neural genes harbor recurrent DNA break clusters in neural stem/progenitor cells. Cell 164:644-655 (2016).

Yurov YB, Iourov IY, Vorsanova SG, Liehr T, Kolotii $\mathrm{AD}$, et al: Aneuploidy and confined chromosomal mosaicism in the developing human brain. PLoS One 2:e558 (2007). 